\title{
Case Study on Screening Emerging Pollutants in Urine and Nails
}

Andreia Alves, ${ }^{\dagger \dagger} \ddagger \nabla_{\odot}$ Georgios Giovanoulis, ${ }^{\S, \|, \nabla_{\odot}}$ Ulrika Nilsson, $"$ Claudio Erratico, ${ }^{\ddagger}$ Luisa Lucattini, ${ }^{\perp}$ Line S. Haug, ${ }^{\#}$ Griet Jacobs, ${ }^{\dagger}$ Cynthia A. de Wit, ${ }^{\|}$Pim E. G. Leonards, ${ }^{\perp}$ Adrian Covaci, ${ }^{\ddagger}$ Jörgen Magner, $^{\S}$ and Stefan Voorspoels $*^{\dagger}$

\footnotetext{
${ }^{\dagger}$ Flemish Institute for Technological Research (VITO NV), Boeretang 200, 2400 Mol, Belgium

${ }^{\ddagger}$ Toxicological Centre, Department of Pharmaceutical Sciences, University of Antwerp, Universiteitsplein 1, B-2610 Wilrijk, Belgium

${ }^{\S}$ IVL Swedish Environmental Research Institute, SE-100 31, Stockholm, Sweden

"Department of Environmental Science and Analytical Chemistry (ACES), Stockholm University, SE-106 91, Stockholm, Sweden

${ }^{\perp}$ Institute for Environmental Studies, VU University Amsterdam, De Boelelaan 1087, $1081 \mathrm{HV}$ Amsterdam, The Netherlands

\#Domain of Infection Control and Environmental Health, Norwegian Institute of Public Health, Lovisenberggata 8, 0456 Oslo, Norway
}

\section{Supporting Information}

ABSTRACT: Alternative plasticizers and flame retardants (FRs) have been introduced as replacements for banned or restricted chemicals, but much is still unknown about their metabolism and occurrence in humans. We identified the metabolites formed in vitro for four alternative plasticizers (acetyltributyl citrate (ATBC), bis(2-propylheptyl) phthalate (DPHP), bis(2-ethylhexyl) terephthalate (DEHTP), bis(2ethylhexyl) adipate (DEHA)), and one FR (2,2-bis (chloromethyl)-propane-1,3-diyltetrakis(2-chloroethyl) bisphosphate (V6)). Further, these compounds and their metabolites were investigated by LC/ESI-Orbitrap-MS in urine and finger nails

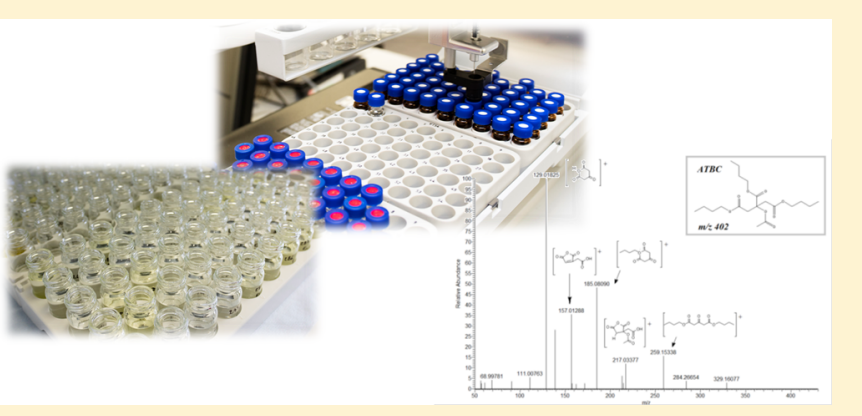
collected from a Norwegian cohort. Primary and secondary ATBC metabolites had detection frequencies (\% DF) in finger nails ranging from 46 to $95 \%$. V6 was identified for the first time in finger nails, suggesting that this matrix may also indicate past exposure to FRs as well as alternative plasticizers. Two isomeric forms of DEHTP primary metabolite were highly detected in urine ( $97 \%$ DF) and identified in finger nails, while no DPHP metabolites were detected in vivo. Primary and secondary DEHA metabolites were identified in both matrices, and the relative proportion of the secondary metabolites was higher in urine than in finger nails; the opposite was observed for the primary metabolites. As many of the metabolites present in in vitro extracts were further identified in vivo in urine and finger nail samples, this suggests that in vitro assays can reliably mimic the in vivo processes. Finger nails may be a useful noninvasive matrix for human biomonitoring of specific organic contaminants, but further validation is needed.

\section{INTRODUCTION}

Recently, new alternative plasticizers have been introduced on the market to replace bis(2-ethylhexyl) phthalate (DEHP), one of the major toxic phthalates used worldwide. DEHP is listed under REACH legislation as a "substance of very high concern" (SVHC), with demonstrated carcinogenic (Group 2B), reproductive toxicity and endocrine disrupting effects on humans. ${ }^{1}$

Alternative plasticizers have been found to migrate out of polymeric products to a lesser extent. ${ }^{2}$ Bui et al. ${ }^{2}$ categorized more than 10 different chemical families of alternative plasticizers (i.e., adipates, citrates, terephthalates, among other). Many of these alternative substances are used in similar applications as phthalates, for example, in toys, vinyl flooring, coated fabrics, gloves, plastic tubing, artificial leather, shoes, sealants, carpet, cosmetic products, medical devices, pharmaceutical tablet coatings, food packaging and beverage closures. ${ }^{2,3}$ For instance, the high molecular weight phthalate bis(2-propylheptyl) phthalate (DPHP) is currently used as a major DEHP substitute. ${ }^{4}$

Limited information is available on the toxicological aspects of the alternative plasticizers, but there are indications of lower toxicity than for DEHP (e.g., no acute toxicity or genotoxicity). However, some recent studies have shown endocrine disrupting potential, developmental and neurological effects and liver carcinogenicity in rats for some of these new chemicals (i.e., for acetyltributyl citrate (ATBC) and bis(2-ethyhexyl) adipate (DEHA) $).{ }^{2,5,6}$ In contrast, other studies did not show any adverse systemic toxicity ${ }^{7}$ or reproductive toxicity in rats $^{8}$ for

Received: November 9, 2016

Revised: January 13, 2017

Accepted: March 15, 2017

Published: March 15, 2017 
some other alternatives (e.g., bis(2-ethylhexyl) terephthalate (DEHTP)).

As described in Bui et al., ${ }^{2}$ humans are exposed to these alternative plasticizers. The estimated risk ratio between the daily intake and the threshold limit for DPHP for infants was 3.4, raising some concern about the use of this plasticizer for example in childcare products and toys. ${ }^{9}$

Another group of chemicals of high concern in regard to human exposure is flame retardants (FRs). Similar to phthalate esters, FRs have been used in a wide range of consumer products (e.g., furniture, textiles, electronic devices, plastics, etc.). ${ }^{10}$ As a consequence of their toxicity, polybrominated diphenyl ether (PBDEs) (e.g., penta-, octa-, and deca-BDE) were banned or restricted from the market ${ }^{11,12}$ leading to demand for new alternative FRs, such as 2,2-bis(chloromethyl)propane-1,3-diyltetrakis(2-chloroethyl) bisphosphate (V6). According to an EU risk assessment report, ${ }^{13} \mathrm{~V} 6$ is primarily used in flexible polyurethane foam and is highly suitable for automotive and furniture applications. Although in the EU report, no acute toxicity to fish, algae and other invertebrates has been observed, possible neurotoxicity and reproductive toxicity were associated with V6 exposure. ${ }^{13,14}$ In fact, V6 is structurally related to the phased-out tris(2-chloroethyl) phosphate (TCEP), ${ }^{15,16}$ which is present as an impurity in the commercial V6 mixture at levels of $4.5-7.5 \% .^{17-19}$ Little is known about V6 toxicity in humans, but studies in dust and air indicate increasing concentrations indoors. ${ }^{17}$ Very high concentrations were found in foam used to produce baby products $\left(>24 \mathrm{mg} / \mathrm{g}\right.$ ) raising concern about human exposure. ${ }^{17}$

Recent biomonitoring studies identified the presence of a few alternative plasticizers, such as DPHP, DEHTP, and DEHA through their oxidative metabolites in urine. ${ }^{4,20-23}$ To our knowledge, the presence of other alternative plasticizers and FRs, such as V6, in urine and/or in other noninvasive matrices such as finger nails has not yet been explored. For urine the exposure is almost immediate depending on the metabolic rate and consequent excretion, while for nails the time window is approximately six months, which is the time it takes for nails to grow out of the nail bed. Therefore, nails have the advantage to indicate long-term exposure. In addition, due to sampling advantages (e.g., cost reduction, less storage, and sample stability), finger nails were considered as an alternative matrix for human biomonitoring (HBM). ${ }^{24-27}$

This study is the first where (1) the metabolites of ATBC, DEHTP, DEHA, DPHP, and V6 identified in in vitro assays were screened in vivo in two noninvasive matrices (finger nails and urine); (2) the elucidation of the new metabolites of alternative plasticizers and FRs (and their fragments) was performed by LC/ESI-Orbitrap-MS; (3) the validity of using urine and finger nails as a noninvasive method to assess exposure to new pollutants was tested in a human cohort.

\section{MATERIALS AND METHODS}

2.1. Reagents and Chemicals. Neat standards for ATBC (98\%), DEHA (99\%), DEHTP ( $\geq 96 \%)$ were obtained from Sigma-Aldrich (Schenelldorf, Germany). Standards for V6 and DPHP were purchased from AccuStandard (Hattersheim, Germany) and Toronto Research Chemicals (Toronto, Canada), respectively. All standard stock solutions (1 mM) were prepared in methanol.

LC/MS-grade methanol and acetonitrile were purchased from Fisher Scientific (Loughborough, UK). The ultrapure water was obtained from a Milli-Q ultrapure water system
(Advantage A10 system, Overijse, Belgium). Trichloroethylene and formic acid (99\%) were supplied by Merck (Darmstadt, Germany). Trifluoroacetic acid (TFA, 99\%) was supplied by Sigma-Aldrich (Steinheim, Germany). Ammonium acetate buffer $\left(\mathrm{NH}_{4} \mathrm{Ac}\right)$ was prepared by dissolving $1.93 \mathrm{~g}$ of ammonium acetate (99.99\% purity, Sigma-Aldrich, Diegem, Belgium) in $100 \mathrm{~mL}$ ultrapure water and acidifying the solution dropwise to $\mathrm{pH} 6.5$ with glacial acetic acid. The enzyme $\beta$ glucuronidase (E. coli K12) was supplied by Roche Applied Sciences (Mannheim, Germany).

2.2. In Vitro Metabolism of ATBC, DEHTP, DEHA, DPHP, and V6. To the authors' knowledge, no authentic standards for most of the metabolites of ATBC, DPHP, DEHTP, DEHA, and V6 (see Supporting Information (SI) Table SI-1) identified in vitro, were commercially available at the time of this study. To bridge this key gap, each compound of interest was individually incubated with human liver/ intestinal microsomes and/or human liver S9 fractions (see SI Figure SI-1). Afterward, the formed metabolites were extracted by liquid-liquid extraction. Positive and negative controls were also prepared to monitor the marker activity of key families of enzymes ${ }^{28-31}$ More details regarding the in vitro generation of the metabolite standards can be found in the SI. These in vitro metabolic extracts were used as reference solutions to create an in house fingerprint mass spectra database for target screening in urine and nail samples.

2.3. Nails and Urine Sampling from a Norwegian Cohort. Details about the sampling are described in Papadopoulou et al. $^{32}$ Finger nail $(N=59)$ and urine (morning) samples $(N=61)$ were collected from 61 volunteers living in the greater Oslo area (Norway). The sampling was approved by the Regional Committee for Medical Research Ethics (REK) in Norway (No. 2013/1269) and before starting the campaign, all participants gave written informed consent to participate. Until analysis all samples were stored at $-20{ }^{\circ} \mathrm{C}$. Before the extraction, residues of nail polish and dirt were cleaned from the nails using acetone, as previously described. $^{25-27,33}$

2.4. Extraction of Alternative Plasticizers and V6 from Nails and Urine. Previously optimized methods were used for extraction of the target plasticizers, V6, and their metabolites in nails and urine. ${ }^{25,26,34}$ For nails, $30 \mathrm{mg}$ of cut pieces were weighed into glass vials followed by extraction of the target analytes using ultrasound-assisted dispersive liquid-liquid microextraction (US-DLLME) as described by Alves et al. ${ }^{25,26}$ Urine samples were extracted following a method involving deconjugation of the phthalate glucuronide, validated by Servaes et al. ${ }^{34}$ Since no internal standards and individual standards of the metabolites were commercially available, only qualitative analyses (screening) were possible. Similarly to Giovanoulis et al. $^{33}$ in which the same urine and finger nail sample extracts were used for the quantification of phthalate and DINCH metabolites, field $\left(n_{1}=10\right)$ and procedural blanks $\left(n_{2}=5\right)$ were analyzed for each extraction method without any phthalate contamination problems. ${ }^{25,26,31}$

2.5. LC/ESI-Orbitrap-MS Analysis. For analysis, an LC (ThermoFisher Scientific, Bremen, Germany) equipped with an Accela quaternary pump, a photodiode array detector (PDA) and a CTC PAL autosampler (CTC Analytics, Zwingen, Switzerland) was used. Chromatographic separations was done on a $\mathrm{C}_{18}$ column $(100 \times 2.1 \mathrm{~mm}, 1.7 \mu \mathrm{m})$ from Phenomenex Inc. (Torrance, $\mathrm{CA}$ ) maintained at $40{ }^{\circ} \mathrm{C}$. The mobile phases used for elution of the target analytes were water (A) and 
$\mathrm{MeOH}$ (B), both buffered with $0.1 \%$ formic acid, following the elution gradient of B: initially $10 \%$; at $9 \mathrm{~min} 60 \%$ keeping conditions constant until $13.6 \mathrm{~min}$; $13.6-15 \mathrm{~min} 80 \%$ keeping conditions constant until $15.5 \mathrm{~min} ; 15.5-20.4 \mathrm{~min} 10 \%$. The flow was kept at $0.3 \mathrm{~mL} / \mathrm{min}$ and the injection volume was 7 $\mu \mathrm{L}$.

For accurate mass measurements, an Orbitrap mass spectrometer (Q-Exactive; Thermo Fisher Scientific, Bremen, Germany) equipped with an ESI source working in polarity switching mode was used (one analysis per mode). Operation parameters were as follows: source voltage, $2.5 \mathrm{kV}$; sheath gas, 53 (arbitrary units); auxiliary gas, 14 (arbitrary units); sweep gas, 3 (arbitrary units); and capillary temperature, $350{ }^{\circ} \mathrm{C}$.

Both in vitro metabolite extracts and human nail and urine samples were analyzed in product ion scan followed by selective ion fragmentation (data dependent $\mathrm{MS}^{2}$ or $\mathrm{ddMS}^{2}$ ) in positive and negative mode (one analysis per mode) to obtain additional structural information. (resolving power set at $17500 \mathrm{fwhm}$, stepped collision energy 10, 30, $50 \mathrm{~V}$, isolation window: $1 \mathrm{~m} / \mathrm{z}$ ).

Data were analyzed using the XCalibur software v.2.2 (ThermoFisher Scientific). The accurate identification of the suspect analytes was performed using a mass deviation $<2.5$ ppm from the target accurate mass. ${ }^{35}$

\section{RESULTS AND DISCUSSION}

3.1. Screening of in Vitro Metabolites of ATBC, DEHTP, DEHA, DPHP, and V6 in Nail and Urine Samples.

From the in vitro experiments a set of 13 metabolites were selected for further identification in the urine and nails using high-resolution LC/ESI-Orbitrap-MS (metabolites in the in vitro extracts are shown in SI Table SI-2). Most of the parent compounds, except for ATBC and V6, could not be efficiently ionized by ESI (neither in positive or negative mode), therefore their presence was not investigated in this study. Other methods such as HPLC/UV or GC/MS may be more suitable for the analyses of these plasticizers. ${ }^{3,21}$

Analysis of procedural blanks revealed that low levels of both mono(2-ethylhexyl) terephthalate (MEHTP) and mono-2ethylhexyl adipate (MEHA) were formed as artifacts during the cleanup step (chemical hydrolysis). However, the (in vivo) enzymatic formation of these metabolites was the predominant pathway, with high peak areas obtained in the blank corrected samples than in the blanks itself (i.e., increase up to $181 \%$ and $89 \%$ peak area of MEHTP isomers and up to $92 \%$ peak area of MEHA in nails and urine, respectively, compared to the peak areas measured in the blanks).

The identified analytes and their detection frequencies (\% DF) in finger nail and urine samples are displayed in Table 1. ATBC and V6 could be identified in finger nails and several metabolites of ATBC, DEHTP, and DEHA were identified in both matrices. No metabolites of DPHP or V6 were detected in any of the analyzed human samples.

In the following sections, levels of detected contaminants and their metabolites are presented and discussed individually.

3.1.1. ATBC. ATBC was detected only in nails samples (49\% $\mathrm{DF}$, Table 1). The quasi-molecular ions corresponding to $[\mathrm{M}+$ $\mathrm{H}]^{+}(m / z 403)$ and $[\mathrm{M}+\mathrm{Na}]^{+}(m / z 425)$ were detected (SI Figure SI-2). In the ddMS ${ }^{2}$ profile, the precursor ion $[\mathrm{M}+\mathrm{H}]^{+}$ was completely fragmented to yield the ions $\mathrm{m} / z \quad 129$ $\left(\mathrm{C}_{5} \mathrm{H}_{5} \mathrm{O}_{4}\right), m / z 157\left(\mathrm{C}_{6} \mathrm{H}_{5} \mathrm{O}_{5}\right), m / z 185\left(\mathrm{C}_{9} \mathrm{H}_{13} \mathrm{O}_{4}\right)$ and $m / z$ $217\left(\mathrm{C}_{8} \mathrm{H}_{9} \mathrm{O}_{7}\right)$. The fragment ions were in agreement with
Table 1. Detection Frequencies (\% DF) of ATBC, DPHP, DEHTP, DEHA, V6 and Their Major in Vitro Metabolites in Finger Nail $(n=59)$ and Urine $(n=61)$ Samples Collected from the Norwegian Participants ${ }^{a}$

\begin{tabular}{|c|c|c|c|c|}
\hline \multirow[b]{2}{*}{ compound } & \multicolumn{2}{|r|}{ nails } & \multicolumn{2}{|r|}{ urine } \\
\hline & $\% \mathrm{DF}$ & $\Delta p \mathrm{pm}^{d}$ & $\% \mathrm{DF}$ & $\Delta p \mathrm{pm}^{d}$ \\
\hline ATBC & 49 & -1.623 & $\mathrm{ND}$ & \\
\hline ATBC-M1, M3 & 46 & -2.028 & ND & \\
\hline ATBC-M2 & 49 & -2.131 & $\mathrm{ND}$ & \\
\hline ATBC-M1b, M2a & 95 & 0.309 & 3 & 1.728 \\
\hline ATBC-M2b & $\mathrm{ND}$ & & NC & \\
\hline $\begin{array}{l}\text { ATBC- } \\
\text { M1e,M2c,M3b }\end{array}$ & ND & & ND & \\
\hline ATBC-M4b & $\mathrm{NC}$ & & $\mathrm{NC}$ & \\
\hline MPHP & $\mathrm{ND}$ & & ND & \\
\hline OH-MPHP & ND & & $\mathrm{ND}$ & \\
\hline MEHTP $^{b}$ & $75 ; 22$ & $-1.274 ;-1.489$ & $97 ; 97$ & $-2.062 ;-2.277$ \\
\hline DEHTP-M2 & ND & & ND & \\
\hline OH-MEHTP ${ }^{c}$ & $8 ; 8$ & $1.068 ; 1.272$ & $\mathrm{ND}$ & - \\
\hline MEHA & 63 & 1.040 & 11 & 0.496 \\
\hline МЕННА & 31 & 1.731 & 98 & 2.463 \\
\hline V6 & 12 & 0.046 & ND & \\
\hline V6-M1, M2 & $\mathrm{ND}$ & & ND & \\
\hline V6-M3 & $\mathrm{ND}$ & & ND & \\
\hline V6-M1b, M2b & ND & & $\mathrm{ND}$ & \\
\hline
\end{tabular}

${ }^{a}$ ND-Not detected; NC-Presence not totally confirmed in finger nails/ urine (deviation in the retention time and fragmentation profile obtained in ddMS ${ }^{2}$ not identical to the in vitro extracts). ${ }^{b}$ Two isomers were identified at 14.9 and $15.1 \mathrm{~min} .{ }^{c}$ Two isomers were identified at 12.1 and $12.3 \mathrm{~min} .{ }^{d}$ Deviation from the target accurate mass (in ppm units) of the $[\mathrm{M}+\mathrm{H}]^{+}$or $[\mathrm{M}-\mathrm{H}]^{-}$ion measured in the nail/urine samples which was used to confirm the identity of the studied compound (i.e., presented the highest levels).

those from ATBC standard. A spectrum of ATBC detected in a finger nail samples is shown in Figure 1.

Recent studies have reported that ATBC has replaced not only DEHP, but also di- $n$-butyl phthalate (DnBP) and butylbenzyl phthalate (BBzP), due to new cosmetics regulations. $^{36}$ ATBC is now used in a wide number of applications, such as additive in cosmetics (mostly in nail lacquer), food packaging and as a flavoring substance in food. ${ }^{37-39}$ Johnson $(2002)^{37}$ suggested that after its absorption, ATBC is mainly excreted via urine/feces in less than $48 \mathrm{~h}$ (in rats). The identification of ATBC in finger nails implies either that it is retained for a longer time in the body than expected (either in blood/serum or in the nail plate), or that there is ubiquitous exposure. However, it is not yet clear how ATBC accumulates in nails, nor which mechanism is favored (i.e., via blood/serum, by deposition in the nail plate or both). Therefore, it is difficult to state whether ATBC levels measured in nails are coming from internal or external exposure. Thus, further investigations are needed to clarify this.

Two chromatographic peaks could be attributed to the previously identified ATBC metabolites, namely ATBC-M1,M3 (46\% DF) and ATBC-M2 (49\% DF). The shorter retention on the $\mathrm{C}_{18}$ stationary phase for those compounds compared with that of ATBC is in accordance with compounds of higher hydrophilicity, such as oxidative metabolites. MS spectra and suggested structures for the fragments are shown in SI Figures SI-3 and SI-4.

ATBC-M1b, M2a were detected in $95 \%$ of the finger nail samples and in $3 \%$ of urine samples. The fragment ions $\mathrm{m} / \mathrm{z}$ 


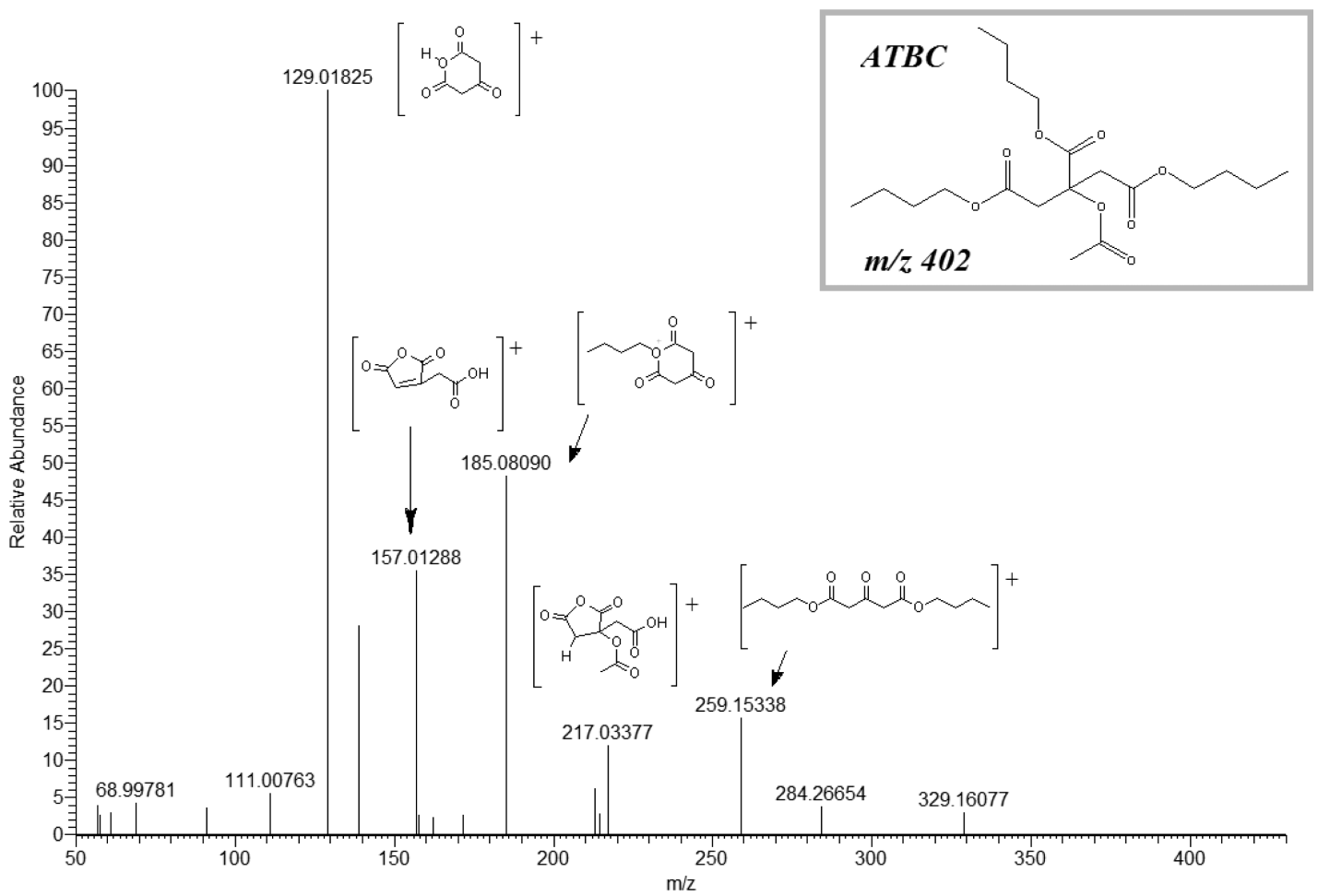

Figure 1. Mass spectrum $\left(\mathrm{ddMS}^{2}, \mathrm{ESI}^{+}\right)$of $\mathrm{ATBC}$ in nails and suggested structures of fragment ions.

185 and $\mathrm{m} / z 211$ were identified in finger nails, as well as in vitro, while $m / z 241$ was only identified in the latter. However, the relative fragment intensities varied significantly between the samples (SI Figure SI-5). As an example, the $[\mathrm{M}-\mathrm{H}]^{-}$ion $(\mathrm{m} / z$ 302) was detected at higher abundance in urine than in the other samples (SI Figure SI-5), suggesting that of one of the isomers (i.e., ATBC-M1b or ATBC-M2a) occurred at a higher concentration in those samples.

Two different metabolic pathways from the isomeric precursors (M1 and M2) could favor the in vivo formation of either ATBC-M1b or M2a (SI Table SI-2). Overall, our data suggest that, when both isomers are formed in vivo, one may be preferentially excreted in urine, whereas the other is rather accumulated (and detected) in nails. Since there are no individual standards for each of the isomers (ATBC-M1b and ATBC-M2a), it is difficult to elucidate which specific isomer is rapidly excreted in urine and/or is accumulated in nails. Even if we assume that the participants had similar exposure patterns over six months in order to compare results from urine and finger nail samples, the complete picture of exposure still remains unclear in order to make such prediction.

Overall, our results indicate that human finger nails may be a suitable matrix for noninvasive monitoring of ATBC exposure, preferably by measuring its primary (M1, M2, and M3) and secondary (ATBC-M1b, M2a) metabolites.

3.1.2. DPHP. No metabolites corresponding to those generated from in vitro experiments with DPHP were detected in finger nails or urine samples. These results support our previous study of human exposure to DPHP and other plasticizers, where three urine spots from the same human study population were analyzed. For DPHP, no metabolites (i.e., MPHP) were detected in the morning urine spot. $^{33}$ MPHP was also investigated in finger nails by Giovanoulis et al. $^{33}$ where it was present in $37 \%$ of the samples. However, in the present study we did not obtain the same result due to the low intensity of MPHP identified in product ion scan.

Some DPHP metabolites were previously identified in urine by Gries et al., ${ }^{40}$ suggesting that the oxidative metabolites, that is, OH-MPHP and two others mono-2-(propyl-6-carboxyhexyl)-phthalate (cx-MPHxP) and mono-2-(propyl-6-oxoheptyl)-phthalate (oxo-MPHP)) are more suitable biomarkers for DPHP exposure. In our study, OH-MPHP was identified as a DPHP metabolite in the in vitro metabolism assays. However, its presence in urine was not confirmed. This is in line with Gries et al. $^{40}$ who found rather low detection frequencies of OH-MPHP (<LOQ-1.25 $\mu \mathrm{g} / \mathrm{L} ; 25 \% \mathrm{DF})$ and oxo-MPHP $(<\mathrm{LOQ}-1.22 \mu \mathrm{g} / \mathrm{L} ; 10 \% \mathrm{DF})$ in human urine. This indicates a low ability to identify DPHP exposure from its metabolites. Since cx-MPHP was not identified as a relevant DPHP metabolite in the in vitro assays, its presence was not investigated further in the urine samples.

Another DPHP metabolite of interest is oxo-MPHP, of which a chromatographic peak at $12.40 \mathrm{~min}$ (lower retention time than OH-MPHP) and a precursor ion $(\mathrm{m} / z$ 319) were observed in the in vitro extracts. However, due to its low intensity in the product ion scan spectrum, no further fragmentation in ddMS ${ }^{2}$ could be obtained. The presence of this metabolite was explored in the urine samples, but it was not detected.

These results are in agreement with Leng et al., ${ }^{4}$ who suggested that MPHP and OH-MPHP are the predominant metabolites over cx-MPHP, and they are rapidly excreted in urine $(<48 \mathrm{~h})$. Also, Schütze et al. ${ }^{20}$ did not detect $\mathrm{cx}-\mathrm{MPHP}$, whereas $\mathrm{OH}-\mathrm{MPHP}$ and oxo-MPHP were present with low detection frequencies $(3-22 \% \mathrm{DF})$ in urinary samples $(\mathrm{n}=$ 300 ) collected over a period of more than 10 years (19992012). These results confirm the low abundance of these metabolites in urine, possibly because of their low rates of formation in vivo, as suggested by our in vitro data. 

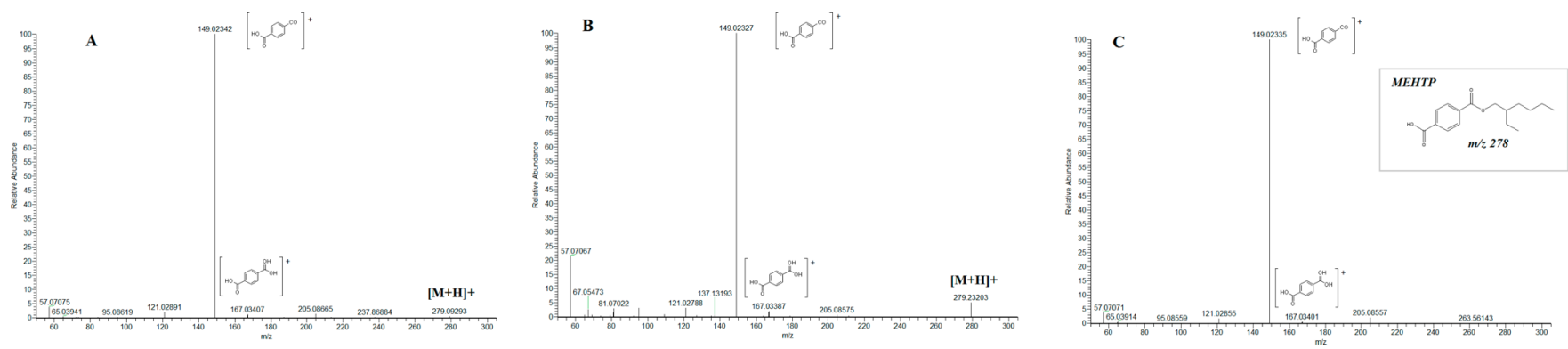

Figure 2. Mass spectra (ESI+) of MEHTP metabolite detected in nails (A), urine (B) and in vitro (C) and suggested structures of fragment ions.
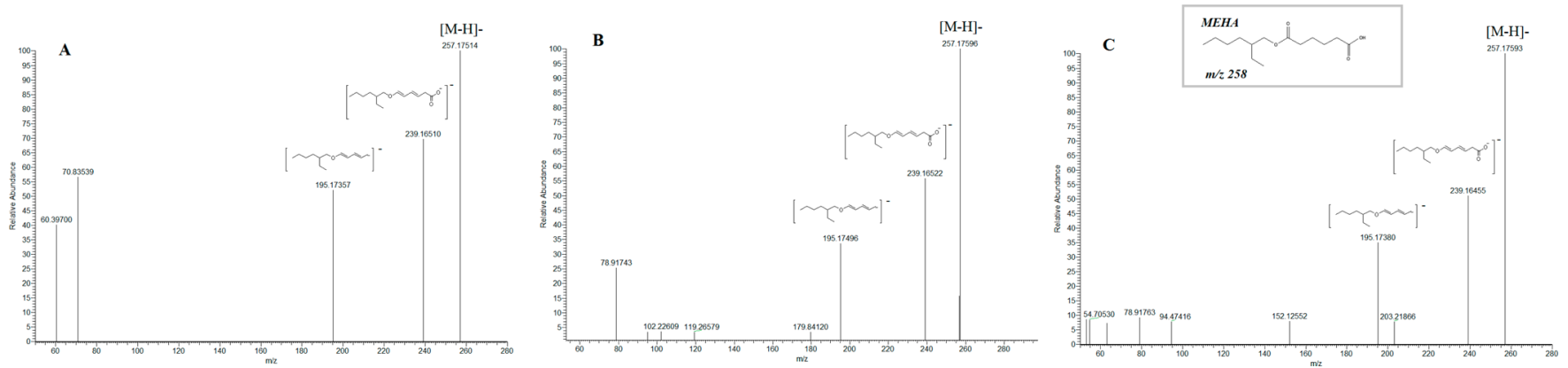

Figure 3. Mass spectra $\left(\mathrm{ESI}^{-}\right)$of MEHA metabolite detected in nails (A), urine (B) and in vitro (C) and suggested structures of fragment ions.

Thus, similar to the excretion of DEHP oxidative metabolites, ${ }^{41}$ DPHP secondary metabolites are excreted rapidly in urine (although relatively low levels are reported). Because of this rapid excretion, finger nails do not seem to be a suitable matrix for DPHP exposure measurements and sensitive (extraction/analytical) tools are needed for measuring DPHP oxidative metabolites in urine.

3.1.3. DEHTP. Two metabolites from DEHTP, namely MEHTP and 1-mono-(2-ethyl-hydroxy-hexyl) benzene-1,4dicarboxylate (OH-MEHTP), were identified in nails and/or in urine (Table 1).

Two isomers of MEHTP were identified in urine and nails with high detection frequencies (97\% and $75 \% \mathrm{DF}$, respectively) for both isomeric forms. The presence of MEHTP was confirmed by the major fragment at $\mathrm{m} / z 149$ $\left(\mathrm{C}_{8} \mathrm{H}_{5} \mathrm{O}_{3}\right)$ in nails, urine and in the in vitro extracts (Figure 2 ). A minor fragment at $m / z 167\left(\mathrm{C}_{8} \mathrm{H}_{6} \mathrm{O}_{4}\right)$ was also present in the spectrum from both matrices and the in vitro metabolism experiment.

Previously, Silva et al. ${ }^{42}$ studied the in vitro metabolism of DEHTP in order to investigate suitable biomarkers of DEHTP exposure. As a result, terephthalic acid (TPA) and several oxidative DETHP metabolites were identified as promising biomarkers in humans. In the present study, the presence of oxidative DEHTP metabolites was also investigated in the metabolism experiments. OH-MEHTP was only detected in finger nails $(8 \% \mathrm{DF})$, for which the characteristic $[\mathrm{M}-\mathrm{H}]^{-}$ion $(\mathrm{m} / z 293)^{42,43}$ was the only present fragment in the spectra from the two observed isomers (SI Table SI-2). As up to eight isomers of monohydroxylated MEHTP are possible, the formation of more metabolites (or different ones) in vivo seems plausible. These findings are in agreement with Silva et $\mathrm{al}^{42}$ who found several OH-MEHTP isomers formed in vitro.

The detection of 1-mono-(2-ethyl-oxo-hexyl) benzene-1,4dicarboxylate (oxo-MEHTP) was investigated. In the in vitro extracts, oxo-MEHTP was detected at relatively low concentrations, suggesting it as a minor DEHTP metabolite. Hence, its presence was not investigated further in the urine and nail samples.

Previously, Lessmann et al. ${ }^{43}$ suggested suitable DEHTP oxidative metabolites as biomarkers of DEHTP in urine. Those included 1-mono-(2-ethyl-5-hydroxy-hexyl) benzene-1,4-dicarboxylate (5-OH-MEHTP); 1-mono-(2-ethyl-5-oxo-hexyl) benzene-1,4-dicarboxylate (5-oxo-MEHTP); 1-mono-(2-ethyl-5carboxyl-pentyl) benzene-1,4-dicarboxylate (5-cx-MEPTP); and 1-mono-(2-carboxyl-methyl-hexyl) benzene-1,4-dicarboxylate (2-cx-MMHTP). 5-cx-MEPTP and 2-cx-MMHTP were not identified in our metabolism study. The $24 \mathrm{~h}$ urinary excretion factors found in that study were 1.72 for 5-OH-MEHTP, 0.95 for 5-oxo-MEHTP, 12.24 for 5-cx-MEPTP and 0.27 for 2-cxMMHTP ( 5.18 total of all four). These can be used to calculate DEHTP intakes based on metabolite concentrations in environmental and occupational studies. ${ }^{22}$ Likewise, in a previous DEHP exposure assessment, ${ }^{42}$ the secondary oxidative metabolites were more representative of internal exposure to the parent phthalate due to the fast metabolism of MEHTP. However, Lessmann et al. ${ }^{22}$ found the levels of the secondary metabolites in the human urine samples to vary significantly, where some were not detected (<LOQ $38.7 \mu \mathrm{g} / \mathrm{L}$ ). Also, in general the \%DF was below $21 \%$, except for the 5-cx-MEPTP, which was the major metabolite ( $94 \%$ DF).

Before this study, none of DEHTP metabolites have been explored in finger nails, therefore our results can suggest this matrix as a possible alternative.

3.1.4. DEHA. All DEHA exposure assessments conducted in humans so far have been performed by measuring the parent compound in urine or breast milk. ${ }^{44-46}$ To our knowledge, the present study is one of the first where metabolites of DEHA have been both generated in vitro and further detected in two different matrices (i.e., urine and finger nails, see Table 1). Previously, Silva et al. ${ }^{23}$ have conducted the in vitro metabolism of DEHA in human liver microsomes, in which MEHA (primary metabolite) and three secondary metabolites (mono2-ethylhydroxyhexyl adipate (MEHHA), mono-2-ethyloxohexyl 
adipate (MEOHA) and adipic acid) were identified and further detected in urine samples $(N=144)$ from American adults. Yet, the authors ${ }^{23}$ did not elucidate or provide a possible explanation for the fragments obtained for each DEHA metabolite.

In our study, MEHA was mainly identified in finger nails, while MEHHA (oxidative) was shown to be mostly present in urine, as shown in Table 1, suggesting that the results from our in vitro experiments were able to partly predict the DEHA metabolism in humans.

For the MEHA identification, neutral losses of $\mathrm{H}_{2} \mathrm{O}$ and $\mathrm{CO}_{2}$ from the quasi-molecular ion are suggested, yielding the fragments at $m / z 239$ and 195, respectively (Figure 3).

The quasi-molecular ion of MEHHA could be identified at $m / z 273$ in spectra from both urine and nails, whereas the ion ratios for the fragment ions differed (Figure SI-6). A reason for that could be that other isomers are formed in vivo than the ones seen in in vitro. MEHHA was detected at a significantly lower frequency in finger nails than in urine, suggesting that its accumulation is rather low. Further investigation is required in order to clarify which of the isomeric forms of MEHHA is mainly formed in vivo (e.g., urine).

3.1.5. V6. V6 has previously been identified in environmental matrices such as dust, which is considered as a relevant pathway of human exposure to both V6 and other flame retardants (e.g., PBDEs). ${ }^{17,47,48}$

Although noninvasive human matrices (i.e., hair and nails) for biomonitoring have been recently used for the investigation of exposure to alternative FRs and organophosphate esters, ${ }^{49,50}$ this is the first time that such samples were analyzed to confirm the presence of V6 exposure. The authors are aware of the current limitations of the study (i.e., same extraction methods were applied as for other environmental organic contaminants without method evaluation), but assessment of the V6 exposure could still be successfully performed. The aim of the present study was only to identify any occurrence of V6 and its metabolites. The metabolites of V6 that were expressed in vitro (and any other plasticizer presented here) are not commercially available, therefore it is a future challenge to produce and isolate such metabolites. Such standards are a must for successful human biomonitoring studies for V6(-metabolites). Notwithstanding these limitations, this study provides new insights to unravel important extraction/analytical parameters in further investigations (e.g., method development). Despite the low detection frequency (12\% DF, Table 1), V6 was identified in nails. This may reflect the low bioaccumulation potential of V6 in this matrix. Nevertheless, there is (1) no indication about the variation in exposure during six months (time that nail takes to completely grow out $)^{51}$ and over the 48 $\mathrm{h}$ period (time between exposure and excretion in urine); (2) lack of knowledge on how V6 is metabolized in the body and transferred to the nail (unknown pharmacokinetics).

Thus, these issues need to be addressed in future research for better understanding of human exposure to V6 and the use of finger nails in biomonitoring studies.

In the analysis, both retention time and spectrum were in accordance with the reference compound of V6 (Figure SI-7). MS/MS spectra from the samples in vivo was not possible to obtain due to low intensities of the precursor ions. However, characteristic fragments at $\mathrm{m} / z 235$ and 297 for V6 were confirmed in ddMS $^{2}$ spectra from in vitro metabolism extracts. $^{18}$

\section{STUDY LIMITATIONS AND FUTURE PERSPECTIVES}

This study indicates that most of the targeted compounds can accumulate in nails. Thus, it is important to highlight that the levels measured in this matrix may reflect contributions from both internal and external exposures, which are difficult to distinguish. There is still a lack of knowledge about their pharmacokinetics in urine and/or serum/blood. Metabolism of the compounds after their incorporation in the nail plate, either from deposition at external exposure, upon penetration in the skin or via blood/serum needs to be deeply investigated, as well as the toxicological effects of the new metabolites. Since standards of the in vitro metabolites from ATBC, DEHTP, DPHP, DEHA, and V6 are not commercially available, it is difficult to quantify them properly. For this study, metabolites generated in in vitro enzymatic assays were formed in small amounts, and therefore used solely for identification on the different matrices. Moreover, the metabolites were generated as a complex mixture, making the analytical identification of individual metabolites even more challenging. Especially for V6, the method used was not validated for quantification purposes and consequently the results are generally indicative of V6 exposure. Developmental of new extraction methods and synthesized pure standards would be a desirable next step for confirmation, as well as for quantification of the target metabolites in vivo.

\section{ASSOCIATED CONTENT}

\section{Supporting Information}

The Supporting Information is available free of charge on the ACS Publications website at DOI: 10.1021/acs.est.6b05661.

Additional information as noted in the text (PDF)

\section{AUTHOR INFORMATION}

\section{Corresponding Author}

*Phone: +32 143350 21; fax.+ 32143194 72; e-mail: Stefan. Voorspoels@vito.be.

ORCID 8

Andreia Alves: 0000-0002-2737-8404

Georgios Giovanoulis: 0000-0003-4473-2345

Author Contributions

${ }^{\nabla}$ A.A. and G.G. contributed equally.

\section{Notes}

The authors declare no competing financial interest.

\section{ACKNOWLEDGMENTS}

We acknowledge the [European Union] Seventh Framework Programme ([FP7/2007-2013] under grant agreement no. [316665] (A-TEAM) for the funding support. Special thanks are also given to Dr. Eleni Papadopoulou and Dr. Juan A. Padilla-Sánchez for their support in the A-TEAM sampling campaign. Dr. Sebastiaan Bijttebier is acknowledged for his support in the LC-Orbitrap measurements.

\section{REFERENCES}

(1) IARC (International Agency for Research on Cancer). Some chemicals present in industrial and consumer products, food and drinking-water: di(2-ethylhexyl) phthalate. IARC Monogr. Eval. Carcinog. Risks Hum. 2012, 101, 149-284.

(2) Bui, T.; Giovanoulis, G.; Palm, A.; Magnér, J.; Cousin, I. T.; De Wit, C. A. Human exposure, hazard and risk of alternative plasticizers to phthalate esters. Sci. Total Environ. 2016, 541, 451-467. 
(3) Radaniel, T.; Genay, S.; Simon, N.; Feutry, F.; Quagliozzi, F.; Barthélémy, C.; Lecoeur, M.; Sautou, V.; Décaudin, B.; Odou, P. Quantification of five plasticizers used in PVC tubing through high performance liquid chromatographic-UV detection. J. Chromatogr. B: Anal. Technol. Biomed. Life Sci. 2014, 965, 158-163.

(4) Leng, G.; Koch, H. M.; Wolfgang, G.; Schütze, A.; Langsch, A.; Brüning, T.; Otter, R. Urinary metabolite excretion after oral dosage of bis (2-propylheptyl) phthalate (DPHP) to five male volunteers Characterization of suitable biomarkers for human biomonitoring. Toxicol. Lett. 2014, 231, 282-288.

(5) Dalgaard, M.; Hass, U.; Vinggaard, A. M.; Jarfelt, K.; Lam, H. R.; Sørensen, I. K.; Sommer, H. M.; Ladefoged, O. Di(2-ethylhexyl) adipate (DEHA) induced developmental toxicity but not antiandrogenic effects in pre- and postnatally exposed Wistar rats. Reprod. Toxicol. 2003, 17, 163-170.

(6) Ghisari, M.; Bonefeld-Jorgensen, E. C. Effects of plasticizers and their mixtures on estrogen receptor and thyroid hormone functions. Toxicol. Lett. 2009, 189, 67-77.

(7) Wirnitzer, U.; Rickenbacher, U.; Katerkamp, A.; Schachtrupp, A. Systemic toxicity of di-2-ethylhexyl terephthalate (DEHT) in rodents following four weeks of intravenous exposure. Toxicol. Lett. 2011, 205, $8-14$.

(8) Furr, J.; Lambright, C.; Wilson, V. S.; Foster, P. M.; Gray, L. E., Jr. A short-term in vivo screen using fetal testosterone production, a key event in the phthalate adverse outcome pathway, to predict disruption of sexual differentiation. Toxicol. Sci. 2014, 140, 403-424.

(9) BfR, DPHP detected in toys: BfR assessing the risk of the softener. http://www.bfr.bund.de/cm/343/dphp-in-spielzeugnachgewiesen-bfr-bewertet-risiko-des-weichmachers.pdf. Bundesinstitut für Risikobewertung; 2011.

(10) ATSDR Toxicological Profile for Phosphate Ester Flame Retardants; U.S. Department of Health and Human Services; 2012.

(11) Regulation (EC), 2006. Concerning the registration, evaluation, authorization and restriction of chemicals ("REACH"). OJ L 396, 1 (30.12.06.).

(12) Dodson, R. E.; Perovich, L. J.; Covaci, A.; Van den Eede, N.; Ionas, A. C.; Dirtu, A. C.; Brody, J. G.; Rudel, R. A. After the PBDE phase-out: a broad suite of flame retardants in repeat house dust samples from California. Environ. Sci. Technol. 2012, 46, 13056-66.

(13) EU, 2007. Risk Assessment Report for 2,2-Bis(Chloromethyl) Trimethylene Bis[bis(2-Chloroethyl) Phosphate] (V6) CAS No.: 38051-10-4. EINECS No.: 253-760-2.

(14) McGee, S. P.; Cooper, E. M.; Stapleton, H. M.; Volz, D. C. Early zebrafish embryogenesis is susceptible to developmental TDCPP exposure. Environ. Health Perspect 2012, 120, 1585-1591.

(15) EU, 2008. European Union. Risk Assessment Report of Tri (2Chloroethyl) Phosphate, 2008. CAS No: 115-96-8 EINECS No: 204-118-5.

(16) World Health Organization. EHC 209: Flame Retardants: Tris(Chloropropyl)Phosphate and Tris-(2- Chloroethyl)phosphate, Geneva, Switzerland, 1998.

(17) Fang, M.; Webster, T. F.; Gooden, D.; Cooper, E. M.; McClean, M. D.; Carignan, C.; Makey, C.; Stapleton, H. M. Investigating a novel flame retardant known as V6: measurements in baby products, house dust, and car dust. Environ. Sci. Technol. 2013, 47, 4449-4454.

(18) Ionas, A. C. Migration of hazardous chemicals to the indoor environment - "Horizon Scanning" for flame retardants present in consumer goods, Ph.D. Dissertation, Antwerp University, Belgium, 2016.

(19) Stapleton, H. M.; Klosterhaus, S.; Keller, A.; Ferguson, P. L.; Van Bergen, S.; Cooper, E.; Webster, T. F.; Blum, A. Identification of Flame Retardants in Polyurethane Foam Collected from Baby Products. Environ. Sci. Technol. 2011, 45, 5323-5331.

(20) Schütze, A.; Kolossa-Gehring, M.; Apel, P.; Schröter-Kermani, C.; Leng, G.; Brüning, T.; Koch, H. M. Bis-(2-propylheptyl)phthalate (DPHP) metabolites emerging in $24 \mathrm{~h}$ urine samples from the German Environmental Specimen Bank (1999-2012). Int. J. Hyg. Environ. Health 2015, 218, 559-563.
(21) Gimeno, P.; Thomas, S.; Bousquet, C.; Maggio, A.-F.; Civade, C.; Brenier, C. Identification and quantification of 14 phthalates and 5 non-phthalate plasticizers in PVC medical devices by GC-MS. $J$. Chromatogr. B: Anal. Technol. Biomed. Life Sci. 2014, 949-950, 99108.

(22) Lessmann, F.; Schütze, A.; Weiss, T.; Langsch, A.; Otter, R.; Brüning, T.; Koch, H. M. Metabolism and urinary excretion kinetics of di (2-ethylhexyl) terephthalate (DEHTP) in three male volunteers after oral dosage. Arch. Toxicol. 2016, 90, 1659-1667.

(23) Silva, M. J.; Samandar, E.; Ye, Xiaoyun; Calafat, A. M. In Vitro Metabolites of Di-2-ethylhexyl Adipate (DEHA) as Biomarkers of Exposure in Human Biomonitoring Applications. Chem. Res. Toxicol. 2013, 26, 1498-1502.

(24) Alves, A.; Kucharska, A.; Erratico, C.; Xu, F.; Den Hond, E.; Koppen, G.; Vanermen, G.; Covaci, A.; Voorspoels, S. Human biomonitoring of emerging pollutants through non-invasive matrices: state of art and future potential. Anal. Bioanal. Chem. 2014, 406, 4063-4088.

(25) Alves, A.; Vanermen, G.; Covaci, A.; Voorspoels, S. Ultrasound assisted extraction combined with dispersive liquid-liquid microextraction (US-DLLME) - a fast new approach to measure phthalate metabolites in nails. Anal. Bioanal. Chem. 2016, 408, 6169-6180.

(26) Alves, A.; Koppen, G.; Vanermen, G.; Covaci, A.; Voorspoels, S. Long-term exposure assessment to phthalates: How do nail analyses compare to commonly used measurements in urine. J. Chromatogr. B: Anal. Technol. Biomed. Life Sci. 2016, 1036-1037, 126-135.

(27) Alves, A.; Covaci, A.; Voorspoels, S. Are nails a valuable noninvasive alternative for estimating human exposure to phthalate esters? Environ. Res. 2016, 151, 184-194.

(28) Davies, H. G.; Richter, R. J.; Keifer, M.; Broomfield, C. A.; Sowalla, J.; Furlong, C. E. The effect of the human serum paraoxonase polymorphism is reversed with diazoxon, soman and sarin. Nat. Genet. 1996, 14, 334-336.

(29) Li, B.; Sedlacek, M.; Manoharan, I.; Boopathy, R; Duysen, E. G.; Masson, P.; Lockridge, O. Butyrylcholinesterase, paraoxonase, and albumin esterase, but not carboxylesterase, are present in human plasma. Biochem. Pharmacol. 2005, 70, 1673-1684.

(30) Negreira, N.; Erratico, C.; van Nuijs, A. L. N.; Covaci, A. In vitro phase I and phase II metabolism of $\alpha$-pyrrolidinovalerophenone $(\alpha$ PVP), methylenedioxypyrovalerone (MDPV) and methedrone by human liver microsomes and human liver cytosol. Anal. Bioanal. Chem. 2015, 407, 5803-5816.

(31) Subrahmanyam, V.; Renwick, A. B.; Walters, D. G.; Young, P. J.; Price, R.; Tonelli, A. P.; Lake, B. G. Identification of cytochrome P-450 isoforms responsible for cis-tradamdol metabolism in human liver microsomes. Drug Metab. Dispos. 2001, 29, 1146-1155.

(32) Papadopoulou, E.; Padila-Sanchez, J. A.; Collins, C. D.; Cousins, I. T.; Covaci, A.; de Wit, C. A.; Leonards, P. E. G.; Voorspoels, S.; Thomsen, C.; Harrad, S.; Haug, L. S. Sampling strategy for estimating human exposure pathways to consumer chemicals. Emerging Contaminants 2016, 2, 26-36.

(33) Giovanoulis, G.; Alves, A.; Papadopoulou, E.; Cousins, A. P.; Schütze, A.; Koch, H. M.; Haug, L. S.; Covaci, A.; Magnér, J.; Voorspoels, S. Biomonitoring exposure estimation to phthalates and DINCH in non-invasive matrices from a Norwegian human cohort. Environ. Res. 2016, 151, 80-90.

(34) Servaes, K.; Voorspoels, S.; Lievens, J.; Noten, B.; Allaerts, K.; Van De Weghe, H.; Vanermen, G. Direct analysis of phthalate ester biomarkers in urine without preconcentration: method validation and monitoring. J. Chromatogr A 2013, 1294, 25-32.

(35) Krauss, M.; Singer, H.; Hollender, J. LC-high resolution MS in environmental analysis: from target screening to the identification of unknowns. Anal. Bioanal. Chem. 2010, 397, 943-951.

(36) Regulation (EC) No. 1223/2009 of the European Parliament and of the Council of 30 November 2009 on cosmetic products (recast), Off. J. Eur. Union L342/59 (2009).

(37) Johnson, W. Final report on the safety assessment of acetyl triethyl citrate, acetyl tributyl citrate, acetyl trihexyl citrate, and acetyl trioctyl citrate. Int. J. Toxicol. 2002, 21, 1-17. 
(38) Technical briefing. Phthalates and Their Alternatives: Health and Environmental Concerns; Lowell Center for Sustainable Production, 2011; pp.1-24.

(39) Maag, J.; Lassen, C.; Brandt, U. K.; Kjølholt, J.; Molander, L.; Mikkelsen, S. H. Identification and assessment of alternatives to selected phthalates, Environmental Danish Ministry of the Environment, Project No. 1341, 2010.

(40) Gries, W.; Ellrich, D.; Küpper, K.; Ladermann, B.; Leng, G. Analytical method for the sensitive determination of major di-(2propylheptyl)-phthalate metabolites in human urine. J. Chromatogr. B: Anal. Technol. Biomed. Life Sci. 2012, 908, 128-136.

(41) Preuss, R.; Koch, H. M.; Angerer, J. Biological monitoring of the five major metabolites of di-(2-ethylhexyl)phthalate (DEHP) in human urine using column-switching liquid chromatography-tandem mass spectrometry. J. Chromatogr. B: Anal. Technol. Biomed. Life Sci. 2005, 816, 269-80 42.

(42) Silva, M. J.; Samandar, E.; Calafat, A. M.; Ye, X. Identification of di-2-ethylhexyl terephthalate (DEHTP) metabolites using human liver microsomes for biomonitoring applications. Toxicol. In Vitro 2015, 29, $716-721$.

(43) Lessmann, F.; Schütze, A.; Weiss, T.; Brüning, T.; Koch, H. M. Determination of metabolites of di(2-ethylhexyl) terephthalate(DEHTP) in human urine by HPLC-MS/MS with on-line clean-up. J. Chromatogr. B: Anal. Technol. Biomed. Life Sci. 2011, 1011, 196-203.

(44) Fromme, H.; Gruber, L.; Schuster, R.; Schlummer, M.; Kiranoglu, M.; Bolte, G.; Völker, W. Phthalate and di-(2-ethylhexyl) adipate (DEHA) intake by German infants based on the results of a duplicate diet study and biomonitoring data (INES 2). Food Chem. Toxicol. 2013, 53, 272-280.

(45) Fromme, H.; Gruber, L.; Schlummer, M.; Wols, G.; Böhmer, S.; Angerer, J.; Mayer, R.; Liebl, B.; Bolte, G. Intake of phthalates and di(2-ethylhexyl)adipate: Results of the Integrated Exposure Assessment Survey based on duplicate diet samples and biomonitoring data. Environ. Int. 2007, 33, 1012-1020.

(46) Palm-Cousins, A.; Remberger, M.; Kaj, L.; Ekheden, Y.; Dusan, B.; Brorstrom-Lunden, E. Results from the Swedish National Screening Programme 2006 - Subreport 1: Phthalates; IVL - Swedish Environmental Research Institute, 2007.

(47) Watkins, D. J.; McClean, M. D.; Fraser, A. J.; Weinberg, J.; Stapleton, H. M.; Sjödin, A.; Webster, T. F. Exposure to PBDEs in the Office Environment: Evaluating the Relationships Between Dust, Handwipes, and Serum. Environ. Health Perspect 2011, 119, 12471252.

(48) Johnson, P. I.; Stapleton, H. M.; Sjodin, A.; Meeker, J. D. Relationships between Polybrominated Diphenyl Ether Concentrations in House Dust and Serum. Environ. Sci. Technol. 2010, 44, 56275632.

(49) Liang-Ying, L.; Salamova, A.; He, K.; Hites, R. A. Analysis of polybrominated diphenyl ethers and emerging halogenated and organophosphate flame retardants in human hair and nails. $J$. Chromatogr A 2015, 1406, 251-257.

(50) Alves, A.; Covaci, A.; Voorspoels, S. Method development for assessing the human exposure to organophosphate flame retardants in hair and nails. Chemosphere 2017, 168, 692-698.

(51) Slotnick, M. J.; Nriagu, J. O. Validity of human nails as a biomarker of arsenic and selenium exposure: A review. Environ. Res. 2006, 102, 125-139. 